\title{
BMJ Open Job morale of physicians in low-income and middle-income countries: a systematic literature review of qualitative studies
}

\author{
Alina Sabitova (D , ${ }^{1}$ Sana Zehra Sajun, ${ }^{1}$ Sandra Nicholson, ${ }^{2}$ Franziska Mosler, \\ Stefan Priebe ${ }^{1}$
}

To cite: Sabitova A, Sajun SZ, Nicholson S, et al. Job morale of physicians in low-income and middle-income countries: a systematic literature review of qualitative studies. BMJ Open 2019;9:e028657. doi:10.1136/ bmjopen-2018-028657

- Prepublication history and additional material for this paper are available online. To view these files, please visit the journal online (http://dx.doi. org/10.1136/bmjopen-2018028657).

Received 09 January 2019 Revised 14 November 2019 Accepted 14 November 2019

A) Check for updates

(C) Author(s) (or their employer(s)) 2019. Re-use permitted under CC BY-NC. No commercial re-use. See rights and permissions. Published by BMJ.

${ }^{1}$ Unit for Social and Community Psychiatry, Queen Mary University of London, London, UK

${ }^{2}$ Institute of Health Sciences Education, Queen Mary University of London, London, UK

Correspondence to

Dr Alina Sabitova;

a.sabitova@qmul.ac.uk

\section{ABSTRACT}

Objectives To systematically review the available literature on physicians' and dentists' experiences influencing job motivation, job satisfaction, burnout, wellbeing and symptoms of depression as indicators of job morale in low-income and middle-income countries. Design The review was reported following Preferred Reporting Items for Systematic Reviews and MetaAnalyses guidelines for studies evaluating outcomes of interest using qualitative methods. The framework method was used to analyse and integrate review findings.

Data sources A primary search of electronic databases was performed by using a combination of search terms related to the following areas of interest: 'morale', 'physicians and dentists' and 'low-income and middleincome countries'. A secondary search of the grey literature was conducted in addition to checking the reference list of included studies and review papers. Results Ten papers representing 10 different studies and involving 581 participants across seven low-income and middle-income countries met the inclusion criteria for the review. However, none of the studies focused on dentists' experiences was included. An analytical framework including four main categories was developed: work environment (physical and social), rewards (financial, non-financial and social respect), work content (workload, nature of work, job security/stability and safety), managerial context (staffing levels, protocols and guidelines consistency and political interference). The job morale of physicians working in low-income and middleincome countries was mainly influenced by negative experiences. Increasing salaries, offering opportunities for career and professional development, improving the physical and social working environment, implementing clear professional guidelines and protocols and tackling healthcare staff shortage may influence physicians' job morale positively.

Conclusions There were a limited number of studies and a great degree of heterogeneity of evidence. Further research is recommended to assist in scrutinising contextspecific issues and ways of addressing them to maximise their utility.

PROSPERO registration number CRD42017082579.
Strengths and limitations of this study

- This study is novel in synthesising qualitative data from all available research on low-income and middle-income countries (LMICs) and provides conclusions based on findings from diverse countries, cultural backgrounds and clinical specialties.

- This study can inform the design of potential interventions and workforce policies and interventions in LMICs; therefore, their clinical utility can be advanced.

- Limited availability and heterogeneity of studies allowed drawing only tentative conclusions.

- This study might be limited conceptually since a small number of studies were eligible.

\section{BACKGROUND}

The crisis in human resources for health has been defined as one of the most severe global health problems ${ }^{1}$ and a major barrier to achieving universal health coverage and building a sustainable health system. ${ }^{2}$ This crisis is especially acute for low-income and middle-income countries (LMICs), many of which suffer from both a shortage and poor devotion of healthcare staff. ${ }^{3}$

Due to the far-reaching effect of job morale, interest in the issue among healthcare staff has increased considerably in recent decades. ${ }^{4}$ First, positive job morale is linked to a greater number of healthcare workers being recruited and retained ${ }^{5}$ which appears to be essential in solving the pressing issue of healthcare staff maldistribution in LMICs. $^{2}$ Second, healthcare staff with positive job morale are more likely to provide higher quality care to patients. ${ }^{6} 7$ Furthermore, improving staff well-being could save healthcare spending by decreasing financial investments in medical education ${ }^{8}$ and lower spending on sickness absence and staff turnover. ${ }^{9}$ 
Despite its importance, there is no universally adopted definition for the concept of job morale nor an agreement on what it constitutes. This could partially explain why research studies aiming to measure job morale are somewhat sporadic. ${ }^{10}{ }^{11}$ Although several authors have tried to investigate job morale as a single entity, ${ }^{512-16}$ they ended up measuring its outcomes or explanatory variables. ${ }^{4}$ Particularly, they referred to the significance of job motivation, job satisfaction, well-being, burnout and depressive symptoms. All these variables can be regarded as indicators of job morale.

Most studies on job morale in healthcare have focused on either nurses ${ }^{1011 \text { 17-21 }}$ or healthcare staff in general, ${ }^{51322-25}$ although job morale has been shown to vary by professional group ${ }^{22}$ and training status. ${ }^{26-28}$ A limitation of the current academic literature is that relatively little is known about physicians' and dentists' experience of job morale in LMICs. ${ }^{29-31}$ There is a lack of detailed description of contextual features and latent influences which could be provided by qualitative research. ${ }^{32}$ Identifying and dentists' experiences that influence job morale may help to create an analytical framework for analysing workforce policies and interventions with clinical and economic benefits.

Against this background, this review aimed to answer for the following research question: Which experiences influence job motivation, job satisfaction, burnout, wellbeing and symptoms of depression as indicators of job morale among physicians and dentists in LMICs?

\section{METHODS}

\section{Search strategy}

A systematic search of electronic databases and grey literature was performed according to the review protocol. The following six electronic databases were searched: Scopus, Pubmed, PsycINFO, Embase, Web of Science and The Cochrane Library up to May 2018. Search terms combined three overlapping areas with key words such as 'morale' OR ‘job motivation' OR 'job satisfaction' OR 'well-being' OR 'burnout' OR 'depression symptoms' AND 'physicians' OR 'dentists' AND 'LMICs' (see online supplementary file 1). Publication bias was reduced by searching conference papers and unpublished literature; hand searches of key journals and reference lists were performed. This review was reported following Preferred Reporting Items for Systematic Reviews and Meta-Analyses (PRISMA) guidelines. ${ }^{33}$

\section{Selection criteria}

Studies were eligible if they assessed any one of the job morale constructs such as job motivation, job satisfaction, well-being, burnout and depression symptoms by using qualitative methods; if at least $50 \%$ of the sample were qualified physicians and/or dentists employed in public healthcare settings or if data about qualified physicians and/or dentists employed in public healthcare settings were provided separately; if at least $50 \%$ of the sample were from the LMICs as defined by World Bank criteria ${ }^{34}$ or data from the country of interest was provided separately. Papers were excluded if more than $50 \%$ of the sample were not yet fully qualified physicians and (or) dentists who were undertaking training at the time of the study (medical students, residents, trainees, registrars or junior physicians), and if they were not written using Latin alphabet, Russian or Kazakh. There was no restriction on the date the studies were conducted. All included articles were inspected independently by a second researcher (SZS) to verify inclusion.

Considering the definitional imprecision of job morale and the different dimensions used to characterise it, we employed an inclusive approach adopting of five indicators of interest, including job motivation, job satisfaction, well-being, burnout and depression symptoms.

\section{Review strategy}

Titles and abstracts of identified articles were exported into EndNote V.X8 and were screened by the first reviewer (AS) in order to exclude irrelevant studies and duplicates. Full-text articles were inspected again for the relevance according to the inclusion criteria. A random sample of $20 \%$ of the articles was independently screened by the second reviewer (SZS) at each stage. Discrepancies were resolved by involving a third reviewer (SP). Mismatches at the full-text screening stage were added up and inter-rater reliability calculated. The level of agreement between AS and SZS was $80 \%$, between AS and SP was $75 \%$.

\section{Data extraction and quality assessment}

Data from each paper, including study details, participant demographics and key results were extracted (see online supplementary file 2). In the case of mixed methods studies, only qualitative findings were extracted. The second reviewer (SZS) ensured the accuracy at this stage by extracting data from $20 \%$ of the included papers. One article written in Portuguese was extracted by involving a native speaker. Methodological quality was assessed using the Critical Appraisal Skills Programme (CASP) for qualitative studies. $^{35}$

\section{Data synthesis and risk of bias assessment}

As part of the framework method, ${ }^{36}$ data from the results sections of included articles were coded in the reviewing software (EPPI-reviewer) and preliminary concepts describing physicians' experiences were defined inductively. Similar concepts were grouped into categories and sub-categories independently by two reviewers (AS, SZS) and were discussed with other researchers (SP, FM, SN) to ensure the range and depth of the coding. The defined categories were then organised in the analytical framework. The framework matrix was used to provide a list of illustrative quotations. Additionally, vote counting ${ }^{37}$ was used as a descriptive tool to indicate patterns across the included studies. We calculated the frequency of defined categories to present how prevalent each category was within the included studies. 


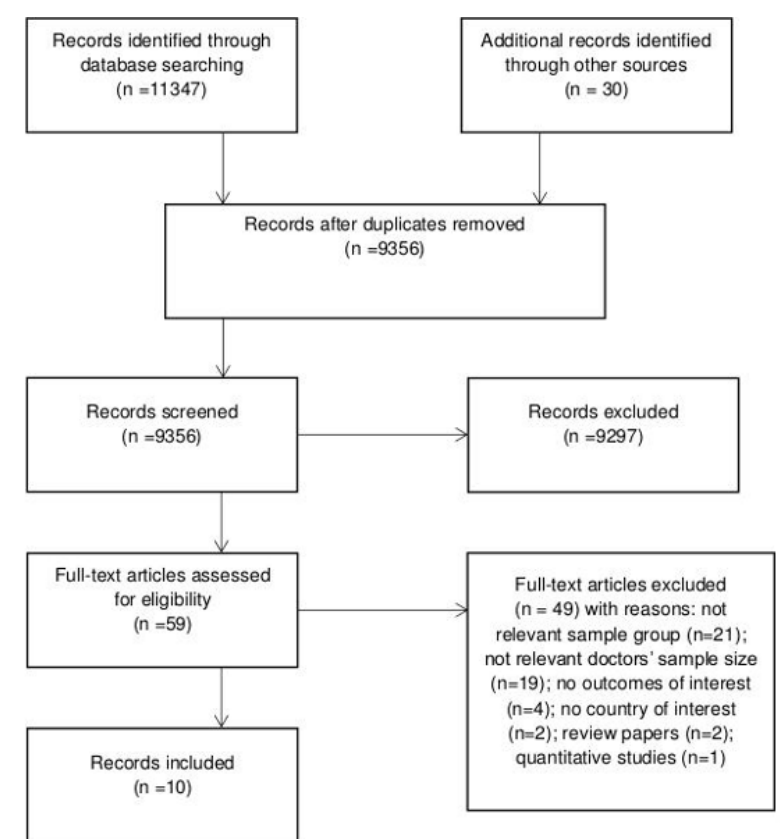

Figure 1 Preferred Reporting Items for Systematic Reviews and Meta-Analyses flow diagram.

Based on CASP studies were appraised in accordance with 10 criteria, where the majority of studies were rated as appropriate with regard to aims, methodology and research findings (see online supplementary file 3 ).

\section{Patient and public involvement}

The results of the analysis were solely based on the previously published literature, as this study did not involve patients or public.

\section{RESULTS}

The original search yielded 11347 articles through database searching and 30 through other sources. A total of 2021 articles were removed as duplicates, and 9297 articles were excluded for not meeting the inclusion criteria. The full texts of the remaining 59 papers were examined, 10 of which were included and represented 10 unique studies. None of the studies focused on dentists' experiences met the inclusion criteria. The detailed selection process is presented in the PRISMA flow diagram (figure 1).

\section{Overview of included studies}

Included studies were published between 2010 and 2017, in English, with the exception of one. They were conducted across seven LMICs, including four uppermiddle-income countries (South Africa, China, Brazil and Russia), two lower-income countries (Pakistan and Moldova) and one low-income country (Uganda). With regard to the study design, four were mixed methods and six were qualitative. The majority of studies were conducted in primary ${ }^{31}{ }^{38-42}$ and secondary healthcare settings. ${ }^{43}$ The included studies' characteristics are summarised in table 1 .

\section{Physicians' experiences influencing job morale}

Identified concepts relevant to physicians' experiences of job morale were grouped into four main framework categories: work environment (I), rewards (II), work content (III) and managerial context (IV). The respective subcategories within each of these categories are presented in the following section. Illustrative quotations within each category are provided in table 2.

\section{Work environment}

Categories such as physical ${ }^{31} 3840-46$ and social ${ }^{31} 3840-46$ work environment appeared in all included studies.

\section{Physical}

Participants expressed that job morale was influenced considerably by working conditions, as a crucial source of job motivation ${ }^{45}$ and satisfaction. ${ }^{38} 40$ Few of them were 'satisfied with physical environment', ${ }^{31}$ but the majority of physicians felt 'very disgusted' ${ }^{46}$ and 'very ashamed ${ }^{44}$ of the hospital infrastructure and constraints of resources, including lack of medicines and equipment deficiency. ${ }^{31} 38404446$ Additionally, physicians noted that poor physical environment in the hospitals 'annoyed patients ${ }^{, 31}$ and showed awareness that poor hygienic conditions were making patients 'more sick'. ${ }^{46}$ The category addressing 'physical work environment' included residential living conditions for physicians who were based in more rural health settings. ${ }^{31} 40$ They described their residences as 'inhabitable' houses with poor 'water and electricity connections', ${ }^{31}$ that are 'falling apart'. ${ }^{40}$ The limited options for schooling for their children ${ }^{31} 46$ and underdeveloped road access ${ }^{31}$ were frustrating and demotivating.

Social

Physicians described a sense of 'collegiality' and 'regular interactions' among staff in the healthcare facilities as a motivator ${ }^{44}$ and perceived 'poor interpersonal relations' as generally as demotivating. ${ }^{45}$ Four main sub-categories contributed to defining the 'social environment' category: relationships with nurses and auxiliary, ${ }^{31} 40414445$ relationships with other physicians ${ }^{40} 44$; relationships with patients ${ }^{31} 3842-44$ and relationships with managers/ supervisors. $^{31} 40434446$

Participants questioned the professional 'competency ${ }^{44}$ and 'power" ${ }^{\text {, }}$ of nurses and noticed that auxiliary staff were 'unsupportive and apprehensive' and worked 'often without a license to practice'. ${ }^{31}$

Relationships with other fellow physicians were found to be "very stimulating, ${ }^{44}$ not only within a hospital, but this view also emerged in case of 'visiting consultants' in rural settings. ${ }^{40}$

There was inconsistency in experiences relating to physician-patient relationships. Some participants 'seemed fairly happy ${ }^{44}$ and 'expressed satisfaction with their current relationships' ${ }^{38}$ However, others expressed the view that physicians 'often had to see angry patients', ${ }^{31}$ who 'could not understand the physicians' work' ${ }^{38}$ 







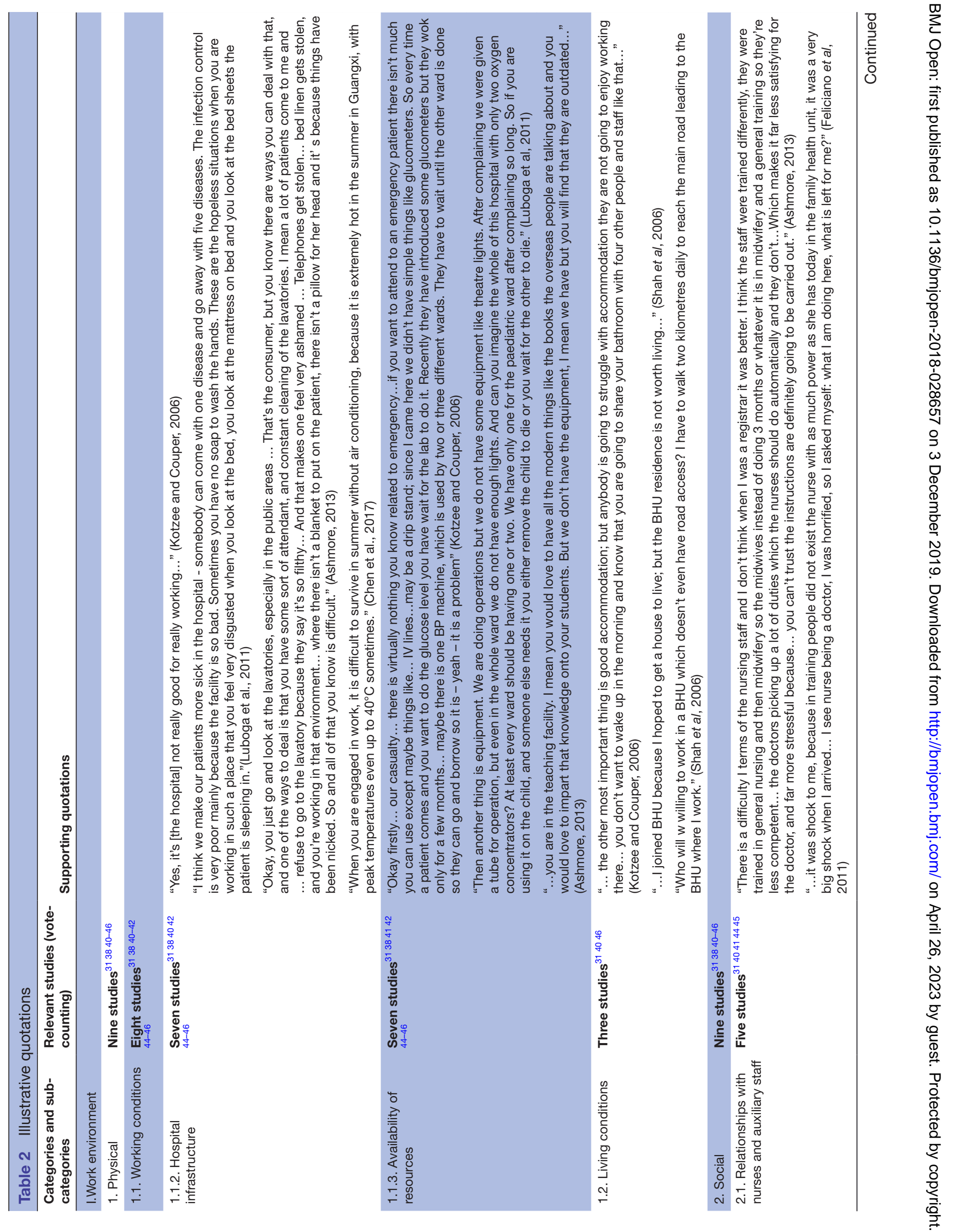




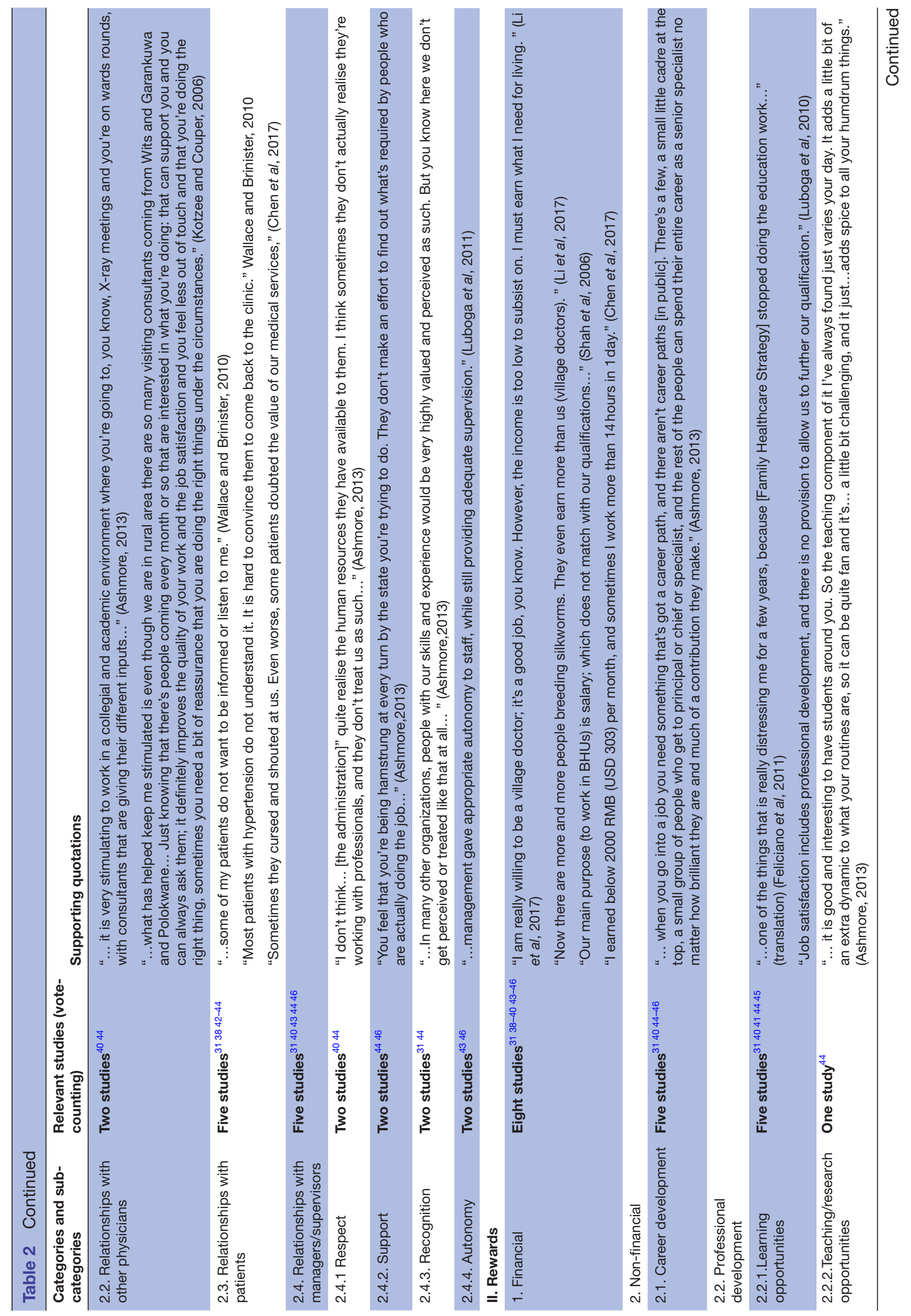




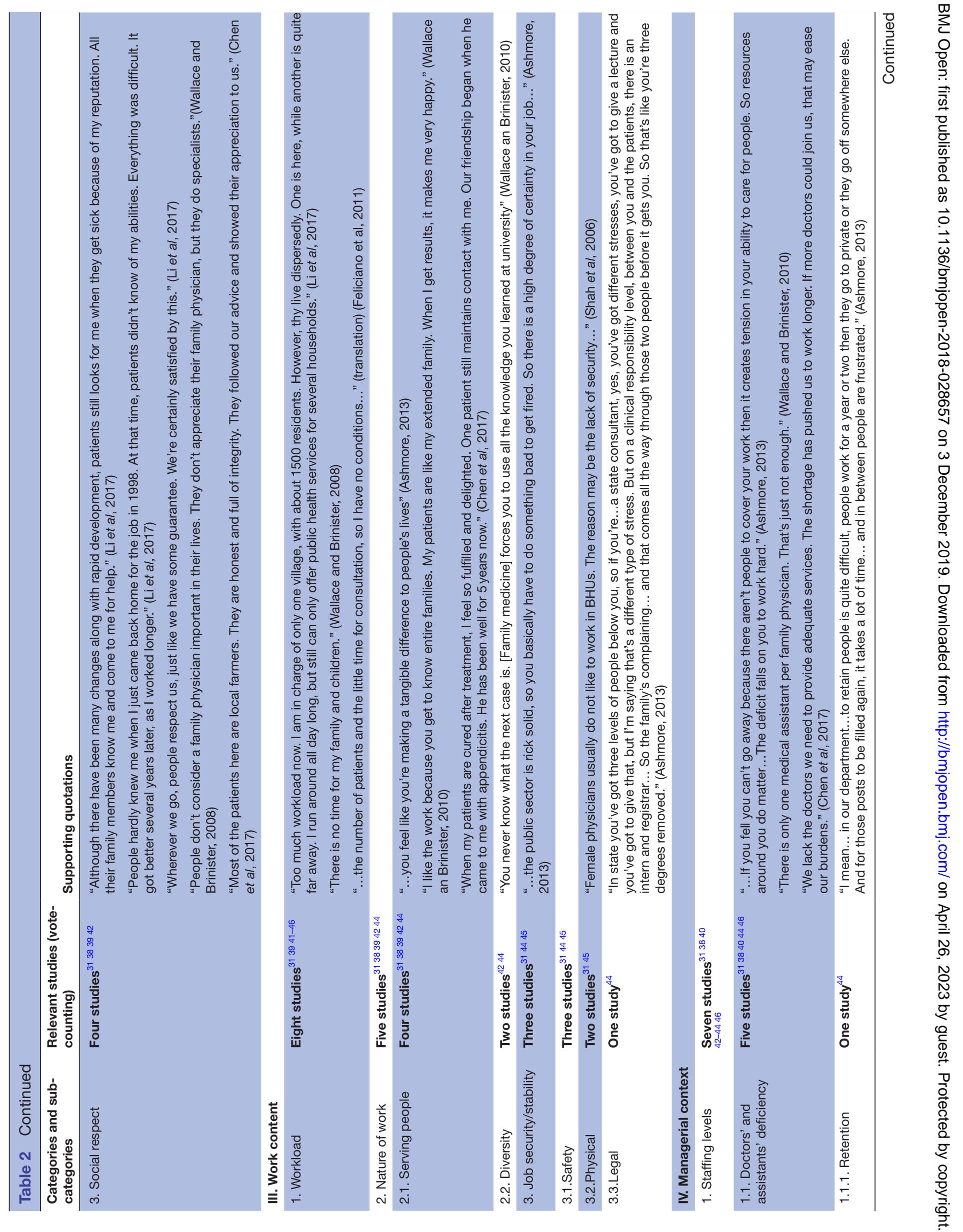




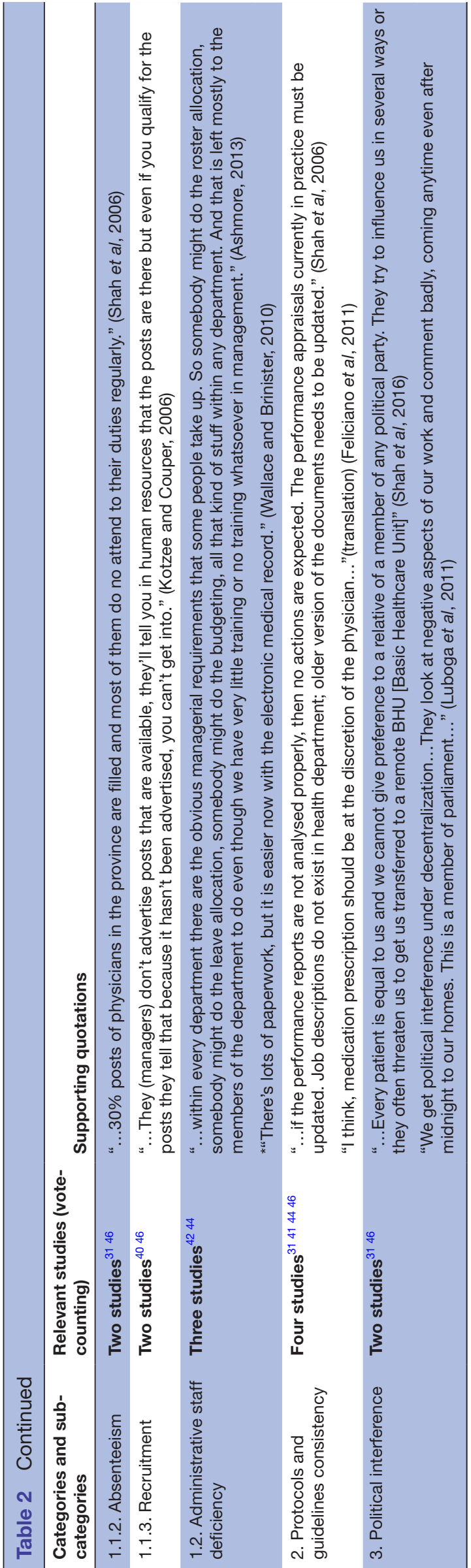

and tend to "bring all their problems [beyond healthrelated] '. ${ }^{42}$ It was emphasised that "difficult" patients are a significant cause of physicians' burnout.

Physicians indicated that relationships with managers/ supervisors mainly depended on the provision of 'adequate supervision ${ }^{46}$ with enough respect, ${ }^{40}{ }^{44}$ support, ${ }^{44} 46$ recognition ${ }^{31} 44$ and autonomy. ${ }^{43} 46$ 'Poor supervision, ${ }^{, 45}$ demotivated physicians and 'total control' by managers/ supervisors contributed to their burnout. ${ }^{43}$

\section{Rewards}

Almost all papers discussed the importance of financial $^{31} 38-4043-46$ and non-financial ${ }^{3138-40}{ }^{44-46}$ rewards in medical practice.

\section{Financial}

The majority of physicians felt that their financial compensation was 'not acceptable', "low' ${ }^{43}$ and 'failed to reflect the job's value', ${ }^{38}$ especially in rural areas ${ }^{39} 40$ and considered their low salaries as a significant 'demotivator' ${ }^{45}$ However, some participants noted that medical practice has advantageous financial incentives, such as state pension, paid holidays and sabbatical leaves. ${ }^{44}$

\section{Non-financial}

Despite the importance of financial incentives, physicians highlighted that "money is not the most important factor for any clinician'. ${ }^{40}$ Career development appeared to be significant in determining physicians' job morale. ${ }^{31} 4044-46$ However, they showed the general sense of dissatisfaction 'with overall process of promotions and transfers in the public health sector'. ${ }^{31}$ Conceptually, career development closely connected with the availability of learning, teaching and research opportunities ${ }^{31} 40414445$ which were 'necessary for the professional growth of physicians'. ${ }^{31}$ Moreover, social respect was also considered a nonfinancial incentive 31383942 which varied in terms of the professional reputation, gained by years of practice ${ }^{39}$ and admiration of public servants, as a part of the community culture $^{31}$ and across different physicians' specialties. ${ }^{42}$

\section{Work content}

The overarching category of 'work content' subcategories, such as workload, nature of work, ${ }^{31} 394244$ job security $^{3144} 45$ and physical and legal safety, was observed in almost all included papers as experiences influencing job morale.

\section{Workload}

The workload was mentioned broadly across all included studies. ${ }^{31}{ }^{39} 41-46$ Specifically, physicians complained about 'too many working hours' ${ }^{43}$ and the necessity to be 'on the end of the phone'. ${ }^{44}$ Emergency duties and long working hours were especially discouraging for married female physicians and single mothers ${ }^{44}$ because they worried that 'their other responsibilities remain unattended'. ${ }^{31}$ Additional frustration was related to a large number of patients in-charge ${ }^{39}$ and 'fixed times for appointments'. ${ }^{42}$ 
Nature of work

Despite the excessive workload, physicians have emphasised that the 'serving' nature of medical profession $^{3138394244}$ and the diversity ${ }^{424}$ of work was extremely satisfying ${ }^{38}$ and motivating. ${ }^{45}$ Participants felt 'a sense of achievement" ${ }^{38}$ when they 'get results and see patients feeling better'. ${ }^{42}$ They also expressed a 'passion to serve their own communities'. ${ }^{31}$

\section{Job security/stability}

Furthermore, some physicians reported that regardless of 'whether you do it well or whether you don't do it so well' ${ }^{44}$ working in public healthcare facilities 'ensured job security for the rest of their careers ${ }^{, 31}$ and provided them with the 'ability to support' their families. ${ }^{45}$

\section{Physical and legal safety}

The motivation experienced as a result of job security and stability was contrasted with the demotivation felt due to low levels of "personal safety', ${ }^{45}$ especially for rural female physicians $^{31}$ and growing responsibility for patients, 'in [a] legal sense'. ${ }^{44}$ However, it has been noted that medico-legal risk for physicians could be mitigated by interns, residents and registrars, who 'shield' physicians from assuming complete medicolegal responsibility for all patients. ${ }^{44}$

\section{Managerial context}

Experiences within the managerial aspect of medical practice were broadly discussed in terms of the staffing levels, ${ }^{31} 384042-44 \quad 46$ protocols and guidelines consistency, $^{31414446}$ and political interference. ${ }^{3146}$

\section{Staffing levels}

Low staffing levels of physicians, medical assistants and managers appeared to be a substantial cause of dissatisfaction $^{3844}$ and contributed towards absenteeism ${ }^{3146}$ and retention problems. ${ }^{44}$ Excessive workload caused by the deficit of physicians ${ }^{46}$ and medical assistants ${ }^{42}$ resulted in physicians being frequently 'absent' from their duties ${ }^{31}$ and 'encourage[d] others to leave' ${ }^{44}$ as well. Moreover, it seemed quite difficult to attract people to work in healthcare facilities, 'despite the district posting the growing vacancies for multiple years, no applications had been received'. ${ }^{46}$ At the same time, physicians raised a concern that vacant posts may not be advertised properly. ${ }^{40}$ The additional burden of paperwork ${ }^{42}$ fell on physicians as a result of administrative staff deficiency ${ }^{44}$ which could be alleviated by implementing electronic medical systems. ${ }^{42}$

\section{Protocols and guidelines consistency}

Physicians stated that job description, protocols and guidelines regulating the drug prescriptions ${ }^{41}$ and performance appraisal ${ }^{31}$ processes "needed to be revised to include the solutions to the current work place problems'. ${ }^{31}$ Nonetheless, the 'growing requirements ${ }^{, 43}$ as a consequence of the increasing number of 'regulations and rules ${ }^{44}$ were highlighted as a source of frustration ${ }^{44}$ and burnout. ${ }^{43}$
Political interference

Certain physicians felt that managerial work context was possibly disrupted by 'politically powerful persons, 31 interfering 'in the decision making [process] at health facilities ${ }^{, 46}$ and their attempts to get a prioritised treatment for relatives. ${ }^{31}$ Some participants believed that it was difficult to be promoted or transferred to a desired position 'without links with any influential person'31 and mentioned cases of 'intimidation of health workers by local politicians'. ${ }^{46}$

\section{DISCUSSION \\ Main findings}

The aim of our systematic review was to synthesise qualitative studies exploring physicians' experiences influencing job motivation, job satisfaction, burnout, well-being and symptoms of depression as indicators of job morale in LMICs.

The analytical framework that comprised four main categories of the work environment (I), rewards (II), work content (III) and managerial context (IV), was developed based on concepts that emerged from included studies. According to the vote counting results, workloads, working conditions and financial rewards were most frequently mentioned as influencing job morale and have been described in almost all studies. The majority of studies mentioned important experiences regarding staffing levels, career and professional development, relationships with nurses/auxiliary staff and managers/supervisors. Physicians from almost half of the included studies focused their attention on the nature of work, relationships with patients, protocols and guidelines consistency.

Physicians were quite consistent in defining whether their experiences were positive or negative. Experiences of excessive workload, low salaries, poor working and living conditions, fewer opportunities for career and professional development, staff shortage, tense physiciannurse and physician-manager/supervisor relationships, inconsistent professional guidelines and political interference were described as negative. Although physicians reported more negative experiences, positive experiences were also underlined in terms of the serving nature of work, being given social respect, job stability and collegial relationships with other physicians.

\section{Strengths and limitations}

To our knowledge, this is the first systematic review of qualitative studies exploring physicians' experiences influencing job morale in LMICs. A further strength is that the review searched through papers from all LMICs and was not limited by physicians' specialty or to English language publications. This allowed for the inclusion of data from diverse countries, cultural backgrounds and clinical specialties. However, this approach presented some limitations. First, although it was possible to extract general concepts in physicians' experiences, there is not enough evidence to assess whether these apply to all 
medical specialties and to other countries. There may be regional and clinical nuances that have not been identified in this review. Second, the prevalence of negative experiences over positive ones could be caused by a biased focus of studies on exploring difficulties. Third, heterogeneity of studies due to imprecise definitions of the concept of 'job morale', made it challenging to provide firm conclusions. Although dentists were included in the literature search, none of the studies on dentists met the inclusion criteria; therefore, the results cannot be generalised to them.

Despite these limitations, the current review is a valuable collation of studies and specifies which experiences influence the job morale of physicians.

\section{Comparison with literature from high-income countries}

The present review supports qualitative findings from previous studies that have been conducted in highincome countries (HICs). It is particularly consistent with findings that serving and helping patients, ${ }^{13} 4748$ working on diverse medical cases ${ }^{13224849}$ and healthy relationships with other medical staff ${ }^{1314485051}$ constitute positive experiences and enhances workers' job morale. It supports evidence that excessive workload, ${ }^{1622495052}$ insufficient staffing levels, ${ }^{131651}$ administrative burden ${ }^{162250}$ and poor relationships and understanding between medical staff and managers ${ }^{1316} 50$ influence job morale negatively. In general, the tendency that professionals are more satisfied with the job content than with its structure and management can be observed not only among physicians. It applies also to employees of different occupations.

Contrary to our findings, healthcare staff employed in high-income countries indicated positive experiences regarding the consistency of existing protocols and guidelines, ${ }^{13}{ }^{48}$ relationships with patients ${ }^{475051}$ and opportunities for continuing education. ${ }^{22}$ The review also demonstrated some evidence regarding poor physical environment within healthcare facilities and constraints of resources, as has been recorded previously. ${ }^{131650}$ However, these findings should be interpreted with caution due to their context-dependency. ${ }^{53}$ The context often includes increasing poverty ${ }^{54}$ inequality ${ }^{55}$ and collapsing healthcare systems. ${ }^{56}$ The structural adjustment programmes promoted by international financial institutions and widely implemented across LMICs may influence the context. ${ }^{58-61}$ In particular, the freezing of vacant posts and mandated ceilings on wages can be substantial barriers to recruiting and retaining healthcare staff. ${ }^{556263}$

Quantitative findings from research on healthcare staff working in HICs helped to corroborate the results of this review. Single studies and reviews conducted in HICs also report associations between job morale and factors such as financial rewards, ${ }^{64-68}$ workload, ${ }^{44-66} 68$ recognition, ${ }^{1323}$ support, $^{1623}$ autonomy, ${ }^{2365} 67$ staffing levels, ${ }^{69}$ learning/teaching/research opportunities, ${ }^{64}{ }^{69}$ workload, ${ }^{44-66} 68$ diversity of work, ${ }^{64} 68$ relationships with colleagues, ${ }^{2364656769}$ job security and protocols and guidelines consistency. ${ }^{1667}$ This is consistent with what this review found in LMICs. Despite this consistency, it is not clear as to whether evidence from HICs can be simply transferred to LMICs and the other way around.

\section{Implications for research and practice}

By considering physicians' experiences across seven LMICs, the current review findings suggest that in order to advance current clinical practices by enhancing job morale, interventions and workforce policies should aim at increasing salaries, improving working and living conditions, tackling healthcare staff shortage and excessive workload and providing more opportunities for career and professional development. However, it is very difficult to achieve in resource-scarce settings. Finding the right balance between growing demands and limited resources is a key challenge. A critical approach to healthcare policy with a specific reference to ethics and a range of disciplines in social science are likely to be required to achieve and maintain that balance. ${ }^{70} 71$ Also, findings suggest that professional guidelines, such as job descriptions, performance appraisal and protocols regulating drug prescriptions should be revised and effectively implemented. This may have a potential positive influence on physician-nurse relationships by maximising role clarity.

There are at least four implications for future research. First, in order to generate clear directives for improvements, future research studies should investigate whether job morale is perceived and valued differently by different medical specialties, and the research gap around dentists' experiences should be addressed. Second, the structural and social determinants of job morale of physicians in LMICs should be studied more systematically which requires funding for such research. Third, contextual features should be considered as they might limit the applicability of findings from one healthcare setting and region to another. Fourth, existing interventions and strategies should be assessed rigorously to define implementation requirements, cost-effectiveness and longterm changes.

\section{CONCLUSIONS}

The current review has identified that perceived threats to positive job morale of physicians in LMICs outweigh perceived incentives. It has highlighted several areas in which strategies aiming to improve physicians' job morale in in LMICs may be targeted. However, generalised conclusions are tentative because of the heterogeneity, limited number and inconsistent quality of the existing studies. Future research into physicians' experiences influencing job morale in LMICs should robustly examine contextspecific issues and appropriate ways of addressing them, to ensure that the results can be translated into practical programmes for improving healthcare practice.

Acknowledgements We are deeply grateful to all members of the Unit for Social and Community Psychiatry for their assistance in data analysis. We wish to thank Dr Mariana Pinto da Costa for translating the article in Portuguese. 
Contributors AS and SP designed the study with input from SN. AS conducted the systematic searches of the literature, selected the studies, performed data analysis and drafted the manuscript. SZS ensured the consistency of study selection, data extraction and analysis. FM contributed to the analysis and edited the manuscript. All authors approved the final version of the manuscript.

Funding This study was sponsored by the Kazakhstan Ministry of Education and Science Center forlnternational Programs as part of a PhD studentship.

Competing interests None declared.

Patient consent for publication Not required.

Provenance and peer review Not commissioned; externally peer reviewed.

Data availability statement All data relevant to the study are included in the article or uploaded as supplementary information.

Open access This is an open access article distributed in accordance with the Creative Commons Attribution Non Commercial (CC BY-NC 4.0) license, which permits others to distribute, remix, adapt, build upon this work non-commercially, and license their derivative works on different terms, provided the original work is properly cited, appropriate credit is given, any changes made indicated, and the use is non-commercial. See: http://creativecommons.org/licenses/by-nc/4.0/.

\section{ORCID iD}

Alina Sabitova http://orcid.org/0000-0002-8314-2797

\section{REFERENCES}

1 Aluttis C, Bishaw T, Frank MW. The workforce for health in a globalized context - global shortages and international migration. Glob Health Action 2014;7:23611.

2 WHO. Global strategy on human resources for health: workforce 2030 Geneva. Switzerland: World Health Organisation, 2016. http:// www.who.int/hrh/resources/pub_globstrathrh-2030/en/

3 WHO. The world health report 2006 - working together for health Geneva. Switzerland: World Health Organisation, 2006. http://www. who.int/whr/2006/en/

4 Grieve S. Measuring morale - Does practice area deprivation affect doctors' well-being? 1997.

5 Reininghaus $\mathrm{U}$, Priebe $\mathrm{S}$. Assessing morale in community mental health professionals: a pooled analysis of data from four European countries. Soc Psychiatry Psychiatr Epidemiol 2007;42:237-43.

6 Department of Health. Nhs health and wellbeing: final report. Department of Health, 2009. http://webarchive.nationalarchives. gov.uk/20130124052412/http://www.dh.gov.uk/prod_consum_dh/ groups/dh_digitalassets/documents/digitalasset/dh_108907.pdf

7 Hall LH, Johnson J, Watt I, et al. Healthcare staff wellbeing, burnout, and patient safety: a systematic review. PLoS One 2016;11:e0159015.

8 Mills EJ, Kanters S, Hagopian A, et al. The financial cost of doctors emigrating from sub-Saharan Africa: human capital analysis. BMJ 2011;343:d7031.

9 Chaudhury NHJ. Ghost doctors: absenteeism in Bangladeshi health facilities. The World Bank, 2003.

10 McFadzean F, McFadzean E. Riding the emotional roller-coaster: a framework for improving nursing morale. $J$ Health Organ Manag 2005;19:318-39.

11 Day GE, Minichiello V, Madison J. Nursing morale: what does the literature reveal? Aust. Health Review 2006;30:516-24.

12 Johnsrud L K. Measuring the quality of faculty and administrative Worklife: implications for college and university Campuses 2002.

13 Totman J, Hundt GL, Wearn E, et al. Factors affecting staff morale on inpatient mental health wards in England: a qualitative investigation. BMC Psychiatry 2011;11.

14 Johnson S, Wood S, Paul M, et al. Inpatient mental health staff morale: a national investigation 2011.

15 Gulliver P, Towell D, Peck E. Staff morale in the merger of mental health and social care organizations in England. J Psychiatr MENT health Nurs. England 2003:101-7.

16 Cahill J, Gilbody S, Barkham M, et al. Systematic review of staff morale in inpatient units in mental health settings 2018

17 Callaghan M. Nursing morale: what is it like and why? J Adv Nurs 2003;42:82-9.

18 Cox KB. The effects of unit morale and interpersonal relations on conflict in the nursing unit. J Adv Nurs 2001;35:17-25.

19 Haw MA, Claus EG, Durbin-Lafferty E, et al. [Improving job morale of nurses despite insurance cost control. 1: Organization assessment]. Pflege 2003;16:103-10.

20 Yang K-P, Huang C-K. The effects of staff nurses' morale on patient satisfaction. J Nurs Res 2005;13:141-52.
21 Hagopian A, Zuyderduin A, Kyobutungi N, et al. Job satisfaction and morale in the Ugandan health workforce. Health Aff 2009;28:w863-75.

22 Priebe S, Fakhoury WKH, Hoffmann K, et al. Morale and job perception of community mental health professionals in Berlin and London. Soc Psychiatry Psychiatr Epidemiol 2005;40:223-32.

23 Johnson S, Osborn D, Araya R, et al. Morale in the English mental health workforce: questionnaire survey 2012.

24 Bowers L, Allan T, Simpson A, et al. Morale is high in acute inpatient psychiatry. Soc Psychiatry Psychiatr Epidemiol 2009;44:39-46.

25 Abraham LJ, Thom O, Greenslade JH, et al. Morale, stress and coping strategies of staff working in the emergency department: a comparison of two different-sized departments. Emerg Med Australas 2018;30:375-81.

26 NHS Staff Surveys. Available: http://www.nhsstaffsurveys.com/Page/ 1064/Latest-Results/2017-Results/

27 Dyrbye LN, West CP, Satele D, et al. Burnout among U.S. medical students, residents, and early career physicians relative to the general U.S. population. Acad Med 2014;89:443-51.

28 Panagopoulou E, Montgomery A, Benos A. Burnout in internal medicine physicians: differences between residents and specialists. Eur J intern Med. Netherlands 2006:195-200.

29 Chopra M, Munro S, Lavis JN, et al. Effects of policy options for human resources for health: an analysis of systematic reviews. The Lancet 2008;371:668-74.

30 Mathauer I, Imhoff I. Health worker motivation in Africa: the role of non-financial incentives and human resource management tools. Hum Resour Health 2006;4.

31 Shah SM, Zaidi S, Ahmed J, et al. Motivation and retention of physicians in primary healthcare facilities: a qualitative study from Abbottabad, Pakistan. Int J Health Policy Manag 2016;5:467-75.

32 Victora CG, Habicht J-P, Bryce J. Evidence-Based public health: moving beyond randomized trials. Am J Public Health 2004;94:400-5.

33 Moher D, Liberati A, Tetzlaff J, et al. Preferred reporting items for systematic reviews and meta-analyses: the PRISMA statement. PLOS Med 2009;6:e1000097.

34 World bank open data, 2018. Available: https://data.worldbank.org

35 Critical appraisal skills programme. CASP qualitative, 2018. Available: https://casp-uk.net/wp-content/uploads/2018/01/CASPQualitative-Checklist.pdf

36 Gale NK, Heath G, Cameron E, et al. Using the framework method for the analysis of qualitative data in multi-disciplinary health research. BMC Med Res Methodol 2013;13:117.

37 Popay J, Roberts H, Sowden A, et al. Guidance on the conduct of narrative synthesis in systematic reviews: a product from the ESRC methods programme 2006.

38 Chen Q, Yang L, Feng Q, et al. Job satisfaction analysis in rural China: a qualitative study of doctors in a township Hospital. Scientifica 2017;2017:1-6.

$39 \mathrm{Li} \mathrm{T}$, Lei T, Sun F, et al. Determinants of village doctors' job satisfaction under China's health sector reform: a cross-sectional mixed methods study. Int J Equity Health 2017;16:64.

40 Kotzee TJ, Couper ID. What interventions do South African qualified doctors think will retain them in rural hospitals of the Limpopo Province of South Africa? Rural Remote Health 2006;6:581.

41 Feliciano KVdeO, Kovacs MH, Sarinho SW. [Burnout among Family Healthcare physicians: the challenge of transformation in the workplace]. Cien Saude Colet 2011;16:3373-82.

42 Wallace LS, Brinister I. Women family physicians' personal experiences in the Republic of Moldova. J Am Board Fam Med 2010;23:783-9.

43 Liadova AV, Korkiya ED, Mamedov AK, et al. The burnout among emergency physicians: evidence from Russia (sociological study). Man in India 2017;97:495-507.

44 Ashmore J. 'Going private': a qualitative comparison of medical specialists' job satisfaction in the public and private sectors of South Africa. Hum Resour Health 2013;11:1.

45 Malik AA, Yamamoto SS, Souares A, et al. Motivational determinants among physicians in Lahore, Pakistan. BMC Health Serv Res 2010;10:201.

46 Luboga S, Hagopian A, Ndiku J, et al. Satisfaction, motivation, and intent to stay among Ugandan physicians: a survey from 18 national hospitals. Int J Health Plann Manage 2011;26:2-17.

47 Zwack J, Schweitzer J. If every fifth physician is affected by burnout, what about the other four? resilience strategies of experienced physicians. Academic Medicine 2013;88:382-9.

48 Sofia Kjellström and Gunilla Avby and Kristina Areskoug-Josefsson and Boel Andersson Gäre and MONICA Andersson B. work motivation among healthcare professionals: a study of well- 
functioning primary healthcare centers in Sweden. Journal of Health Organization and Management 2017;31:487-502.

49 Agana DF, Porter M, Hatch R, et al. Job satisfaction among academic family physicians. Fam Med 2017:49:622-5.

50 Reid Y, Johnson S, Morant N, et al. Explanations for stress and satisfaction in mental health professionals: a qualitative study. Soc Psychiatry Psychiatr Epidemiol 1999;34:301-8.

51 Hall L, Johnson J, Heyhoe J, et al. Strategies to improve general practitioner wellbeing: a focus group study 2017;35.

52 Kruse GR, Chapula BT, Ikeda S, et al. Burnout and use of HIV services among health care workers in Lusaka district, Zambia: a cross-sectional study. Hum Resour Health 2009;7:55.

53 Willis-Shattuck M, Bidwell P, Thomas S, et al. Motivation and retention of health workers in developing countries: a systematic review. BMC Health Serv Res 2008;8:247.

54 Mohindra KS. Healthy public policy in poor countries: tackling macro-economic policies. Health Promot Int 2007;22:163-9.

55 Kentikelenis AE. Structural adjustment and health: a conceptual framework and evidence on pathways. Soc Sci Med 2017; 187:296-305.

56 Nolan T, Angos P, Cunha AJLA, et al. Quality of hospital care for seriously ill children in less-developed countries. The Lancet 2001;357:106-10.

57 Travis P, Bennett S, Haines A, et al. Overcoming health-systems constraints to achieve the millennium development goals. The Lancet 2004;364:900-6.

58 Beste J, Pfeiffer J. Mozambique's debt and the International monetary fund's influence on poverty, education, and health. Int $J$ Health Serv 2016;46:366-81.

59 Laurell AC. Three decades of neoliberalism in Mexico: the destruction of Society. Int J Health Serv 2015;45:246-64.

60 Coburn C, Restivo M, Shandra JM. The African Development Bank and women's health: A cross-national analysis of structural adjustment and maternal mortality. Soc Sci Res 2015;51:307-21.
61 Hossen MA, Westhues A. The medicine that might kill the patient: structural adjustment and its impacts on health care in Bangladesh. Soc Work Public Health 2012;27:213-28.

62 Kentikelenis AE, Stubbs TH, King LP. Structural adjustment and public spending on health: evidence from IMF programs in lowincome countries. Soc Sci Med 2015;126:169-76.

63 Thomson M, Kentikelenis A, Stubbs T. Structural adjustment programmes adversely affect vulnerable populations: a systematicnarrative review of their effect on child and maternal health. Public Health Rev 2017;38:13.

64 Van Ham I, Verhoeven AAH, Groenier KH, et al. Job satisfaction among general practitioners: a systematic literature review. Eur $J$ Gen Pract 2006;12:174-80.

65 Scheurer D, McKean S, Miller J, et al. U.S. physician satisfaction: a systematic review. J Hosp Med 2009;4:560-8.

66 Tomljenovic M, Kolaric B, Stajduhar D, et al. Stress, depression and burnout among hospital physicians in Rijeka, Croatia. Psychiatr Danub 2014;26 Suppl 3:450-8.

67 Goetz K, Jossen M, Szecsenyi J, et al. Job satisfaction of primary care physicians in Switzerland: an observational study. Fam Pract 2016;33:498-503.

68 Joyce C, Wang WC. Job satisfaction among Australian doctors: the use of latent class analysis. J Health Serv Res Policy 2015;20:224-30.

69 Janus K, Amelung VE, Gaitanides M, et al. German physicians "on strike"-Shedding light on the roots of physician dissatisfaction. Health Policy 2007;82:357-65.

70 McCoy D. Critical Global Health: Responding to Poverty, Inequality and Climate Change Comment on "Politics, Power, Poverty and Global Health: Systems and Frames". Int J Health Policy Manag 2017;6:539-41.

71 Schrecker T. Interrogating scarcity: how to think about 'resourcescarce settings'. Health Policy Plan 2013;28:400-9. 